Clarissa C. Trois 2

Bárbara C. Frantz 2

Juliana B. Yaluk 2

Carla A. Taroncher 2

Waleska Schneider 2

Lyryss Helena B. Schonel 2

Ronaldo Bordin 3

\section{Prevalência de CAGE positivo entre estudantes de segundo grau de Porto Alegre, Rio Grande do Sul, Brasil, 19941}

\author{
Prevalence of CAGE-positive secondary school \\ students in Porto Alegre, Rio Grande do Sul, \\ Brazil, 19941
}

1 Apresentado no VI Salão de Iniciação Científica, Pró-Reitoria Adjunta de Pesquisa da Universidade Federal do Rio Grande do Sul, 17 a 21 de outubro de 1994

2 Faculdade de Medicina, Universidade Federal do Rio Grande do Sul. Rua Ramiro Barcel os 2.600, térreo, Porto Alegre, RS 90035-003, Brasil.

3 Departamento de Medicina Social, Faculdade de Medicina, Universidade Federal do Rio Grande do Sul.

Rua Ramiro Barcel os 2.600, térreo, Porto Al egre, RS 90035-003, Brasil.
A bstract This study aims to describe the preval ence of CAGE-positive third-year high school students in Porto Al egre, Rio Grande do Sul, in 1994. The sample consisted of 1,171 students: 598 (51\%) from public and 573 (49\%) from private schools. We found reference to previous alcoholic beverage consumption in $93 \%$ of the intervi ewees. The highest prevalence was among male students $(p<0.02)$ and students from private schools $(p<0.03)$. Half of the students reported weekly consumption, usually when going to parties (60\%) and bars (17\%). CAGE positiveness prevalence was $8.3 \%$ overall and $11 \%$ among males $(p<0.02)$. When variables like gender, age, and soci oeconomic status were controlled, there was no statistically si gnificant difference between the kind of school and CAGE status.

Key words Epidemi ology; Public Health; Students; Alcohol Drinking; Adolescence

Resumo Este estudo objetiva descrever a prevalência de positividade do teste CAGE entre estudantes de terceiro ano do segundo grau de Porto Alegre, RS, em 1994. A amostra foi composta por 1.171 estudantes, 598 (51\%) procedentes de escolas públicas e 573 (49\%) de escol as particulares. Encontrou-se referência de consumo prévio de bebidas al coól icas de $93 \%$, com maior prevalência entre os estudantes do sexo masculino $(p<0,02)$ e aquel es provenientes de escol as particulares $(p<0,03)$. Metade dos al unos rel ataram freqüência de consumo semanal, predominantemente em festas (60\%) e bares (17\%). A prevalência de CAGE positivo foi de $8,3 \%$, atingindo $11 \%$ entre os homens $(p<0,02)$. Não se verificou diferença estatisticamente significativa entre tipo de escola e positivi dade ao teste CAGE quando controladas as variáveis sexo, idade e poder de consumo. Palavras-chave Epidemiologia; Saúde Pública; Estudantes; Consumo de Bebidas Ál coólicas; Adolescência 


\section{Introdução}

Hábito difundido e aceito na maioria dos países, a ingesta excessiva de bebidas alcoólicas terminou por configurar o alcoolismo como um dos grandes problemas de saúde pública da atualidade. Apresenta uma prevalência estimada de cerca de $5 \%$ a $10 \%$ entre os adultos (Knapp et al., 1994; Lowinson et al., 1992), sendo mais comum em regiões urbanas, afetando usualmente indivíduos com história de distúrbios familiares e pais alcoolistas, divorciados ou desquitados e pessoas que não estão ligadas a organizações religiosas (Malbergier et al., 1992).

Estudos epidemiológicos realizados na Amé rica Latina apontam taxas de prevalência global de alcoolismo entre $3 \%$ e $23 \%$, associando-o a importantes causas de morbimortalidade, perfazendo $4,9 \%$ de diagnósticos associados ao consumo excessivo de álcool (doenças, homicídios, acidentes). No Brasil, não existem dados disponíveis sobre a prevalência do alcoolismo na população geral, mas estima-se que aproximadamente $90 \%$ das pessoas consumam álcool, com uma prevalência entre a clientela de serviços gerais de saúde de várias regiões do País variando entre 9\% e 58\% (Duncan, 1991). Soibelman \& Luz Jr. (1996), revisando estudos nacionais recentes, enfatizam a relação direta do consumo de bebidas alcoólicas com diversas complicações clínicas, acidentes de trânsito e de trabalho, diminuição da produtividade e indicadores de violência. Estima-se que metade dos acidentes automobilísticos e suicídios ocorram após uso de álcool, e que os al coolistas sejam responsáveis por $10 \%$ das consultas médicas por causas indiretas, por $15 \%$ a $40 \%$ das internações em hospitais gerais e estejam envolvidos em $23 \%$ a $35 \%$ dos suicídios (Malbergier et al., 1992; Fuchs \& Pechanski, 1992). Cabe salientar que os chamados "bebedoresproblema", aqueles que, embora não apresentem nítida dependência ao álcool, têm al gum problema relacionado à sua ingesta, estão envolvidos em $42 \%$ dos acidentes fatais, $67 \%$ dos casos de abuso infantil, $51 \%$ dos estupros e 38\% dos suicídios (Royce, 1981).

Existem poucos estudos epidemiológicos mundiais que enfoquem os adolescentes e, mais especificamente, na faixa etária correspondente aos estudantes secundaristas brasileiros; os existentes podem não corresponder à nossa realidade. Mesmo assim, uma série de autores (Carmona et al., 1986; Sanchéz \& Zavala, 1986; Urzua \& Kaempffer, 1985; Forney et al., 1988; Conroy, 1988) apontam o álcool como a droga psicotrópica de maior uso entre os jo- vens, provavelmente devido à sua disponibilidade e fácil acesso, particularmente entre estudantes secundaristas, constituindo-se, muitas vezes, como espécie de "gatilho" para o posterior envolvimento com outras drogas psicotrópicas ( cocaína, anfetaminas, maconha etc.).

Em nosso meio, os estudos disponíveis em adolescentes são ainda mais escassos. Entretanto, sabe-se que o consumo de álcool nesses grupos é mais freqüente dos 14 aos 15 anos, geralmente entre indivíduos que começaram a beber antes dos 11 anos e relacionado com a presença de pais ou amigos que também são bebedores. A primeira experiência com bebidas acontece em casa e, à medida em que aumenta a idade, 0 adolescente passa a beber mais com os amigos e menos com os pais, ao contrário do que se observa em idades mais precoces (Forney et al., 1988; McLaughlin et al., 1985, Pechansky, 1993).

A escassez de estudos nesta faixa etária deve-se não apenas às barreiras encontradas na abordagem dos adolescentes, mas principalmente à inexistência de metodologia epidemiológica testada, de fácil aplicação, com ampla experiência de uso e voltada à detecção precoce dos problemas relacionados ao álcool. Uma série de testes de triagem foram desenvolvidos para minimizar essas dificuldades entre os adultos, salientando-se o teste CAGE, por ser superior aos exames laboratoriais disponíveis para triagem de alcoolismo (Bernard et al., 1984; Hays \& Spickard, 1987), ser menos intimidativo e de aplicação mais rápida que os demais questionários utilizados, constituindo uma boa opção na triagem de pacientes com problemas relacionados ao uso de álcool (Masur et al., 1985; Magruder-Habib et al., 1993).

Desenvolvido com base em estudo realizado no North Carolina Memorial Hospital (Ewing, 1984), o teste CAGE foi inicialmente validado em hospital psiquiátrico, em sua versão original (Mayfield et al., 1974) e em português (Masur \& Monteiro, 1983), já tendo sido validado em hospital geral em francês (Rueff et al., 1989), além de aplicado em pacientes ambulatoriais (Bushsbaum et al., 1991) e em estudos populacionais brasileiros ( Moreira, 1993; Gigante, 1988). É composto por quatro perguntas sobre o uso de bebidas alcoólicas, colocadas em tal ordem que facilite o uso mneumônico da sigla CAGE, resultante das letras iniciais das palavras-chave contidas em cada uma: "Alguma vez você sentiu que deveria dimimuir a quantidade de bebida ou parar de beber?" (cut down); "As pessoas Ihe incomodam porque reclamam do seu modo de beber?" (annoyed); “Você se sente culpado pela maneira com que costuma beber?" (guilty) e 
“Você bebe pela manhã para diminuir a ressaca ou o nervosismo?" (eye-openner). São considerados CAGE-positivo os entrevistados que responderem afirmativamente a pelo menos duas questões, atingindo sensibilidade e especificidade que variam de $74 \%-88 \%$ e de $83 \%-91 \%$, respectivamente, na população adulta (Mayfield et al., 1974; Masur \& Monteiro, 1983; Bushsbaum et al., 1991).

Este estudo pretende descrever a prevalência de problemas relacionados ao consumo de álcool (aferido pelo questionário CAGE), além de outros parâmetros de consumo, observados entre estudantes do terceiro ano do segundo grau de escolas públicas e particulares da região urbana do Município de Porto Alegre (RS), supondo-se que a prevalência de Teste CAGE positivo seja maior entre aqueles provenientes de escolas particulares do que entre os de escola pública.

\section{Material e métodos}

A população-alvo deste estudo são todos os estudantes de terceiro ano do segundo grau regularmente matriculados nas escolas públicas (estaduais e municipais) e particulares da área urbana de Porto Alegre. Listagem fornecida pela Secretaria de Educação e Cultura do Estado do Rio Grande do Sul (SEC-RS), contendo o respectivo número de alunos por série e escola, apontava a existência de 47 escolas públicas e 41 privadas e um total de 8.800 alunos nesta série em 1994.

Pressupondo-se uma prevalência de 5,2\% de bebedores excessivos entre os estudantes secundaristas de terceiro ano (Oderich et al., 1995), estimou-se uma amostra de 1.141 alunos, com intervalo de confiança de $95 \%$ e o pior resultado admissível igual a 6,4\%. Foram selecionadas 16 escolas para participar do estudo, de forma estratificada por tipo (oito públicas e oito privadas), através de enumeração do total de escolas listadas e posterior sorteio, com auxílio de uma tabela de números aleatórios. Pela listagem da SEC-RS, deveria haver 793 alunos matriculados nas escolas públicas e 844 nas particulares, totalizando 1.673 alunos. Entretanto, consulta às listagens das escolas evidenciou 1.303 alunos efetivamente matriculados, dos quais 1.171 preencheram o instrumento de coleta de dados, 598 ( $88,1 \%$ do total) alunos das escolas públicas e 573 (91,8\% do total) das particulares.

O instrumento utilizado para a coleta de dados consistiu em questionário composto por vinte questões, incluindo dados sócio-demo- gráficos, as quatro questões que compõem o questionário CAGE na sua versão em português (Masur \& Monteiro, 1983) e quatro questões voltadas especificamente ao consumo de álcool. A variável poder de consumo, adotada como uma aproximação da posição sócio-econômica, foi aferida através de um escore médio, definido pela quantidade de bens de consumo duráveis (eletroeletrônicos e veículos automotores) existente no domicílio dos respondentes, adaptada de escala empregada por agência de publicidade, cujo escore delimita as classes de poder de consumo, de A a E (Almeida \& Wieckerhauser, 1992).

O questionário foi aplicado em todas as turmas de terceiro ano do segundo grau de cada uma das escolas que compuseram a amostra, em qualquer turno, mediante autorização da supervisão ou direção das escolas. Todos os alunos presentes no dia da aplicação, independentemente da idade, foram incluídos. A aplicação do questionário em cada uma das turmas foi feita de forma padronizada, sem que fosse revelado o verdadeiro objetivo do mesmo.

Foram excluídos todos os al unos ausentes no dia de aplicação do questionário, não sendo realizada tentativas posteriores de aplicação do instrumento aos mesmos. Estavam ausentes no momento da aplicação 81 (11,9\%) alunos de escola pública e 51 (8,2\%) de escola particular, totalizando 132 (10\%) alunos ausentes, por qualquer motivo, incluindo evasão. Não participaram do estudo duas escolas particulares, uma por negar a realização do estudo em suas dependências (sendo sorteada outra em seu lugar), e outra por apresentar como norma de ingresso a abstinência absoluta ao álcool (o que poderia constituir um potencial viés de aferição).

Alguns questionários apresentaram determinadas questões incorretamente preenchidas, descartadas no processo de análise, mas mantendo-se as informações das questões corretamente preenchidas.

Empregaram-se para análise estatística os testes do Qui-Quadrado, t de Student e análise multivariada para mensurar a associação entre tipo de escola e possibilidade do Teste CAGE, controlando-se para sexo, idade e poder de consumo. Assumiu-se um alfa convencional de $5 \%$ para todas as análises. Por fim, a versão preliminar deste estudo se constituiu em requisito metodológico parcial da disciplina de Epidemiogia Geral (MED518), destinada a acadêmicos de sétimo semestre da Faculdade de Medicina, Universidade Federal do Rio Grande do Sul (UFRGS). 


\section{Resultados}

Foram entrevistados 1.171 estudantes de terceiro ano do segundo grau, com idades variando entre 15 e 49 anos e média de 18,4 anos, com $4 \%$ do total de alunos possuindo mais de 22 anos de idade. As escolas públicas apresentaram uma média de 18,7 anos, contra 16,8 anos nas particulares ( $p \varangle 0,001$ ), notadamente

Tabela 1

Características da amostra.

\begin{tabular}{lrr}
\hline Variáveis & $\mathrm{n}$ & $\%$ \\
\hline Sexo & & \\
$\quad$ Masculino & 687 & 41,8 \\
$\quad$ Feminino & & 58,2 \\
Religião & 909 & \\
$\quad$ Católica & 240 & 79,1 \\
Outras & & 20,9 \\
Tipo de Escola & 598 & \\
Pública & 573 & 51,1 \\
Particular & & 48,9 \\
Já experimentou & & \\
bebida alcoólica? & 1.085 & 93,4 \\
Sim & 77 & 6,6 \\
Não & & 8,3 \\
CAGE & 94 & 91,7 \\
Positivo & 1.040 & \\
Negativo &
\end{tabular}

Obs.: Percentuais calculados sobre o total de casos com valor para cada variável.

Tabela 2

Relação entre sexo, tipo de religião e de escola segundo experiência prévia com bebidas alcoólicas.

\begin{tabular}{|c|c|c|c|c|c|}
\hline \multirow[t]{3}{*}{ Variáveis } & \multicolumn{4}{|c|}{ Já experimentou bebida alcoólica? } & \multirow[b]{3}{*}{$\mathrm{p}$} \\
\hline & \multicolumn{2}{|c|}{ Sim } & \multicolumn{2}{|c|}{ Não } & \\
\hline & $\mathrm{n}$ & $\%$ & $\mathrm{n}$ & $\%$ & \\
\hline \multicolumn{6}{|l|}{ Sexo } \\
\hline Masculino & 461 & 95,4 & 22 & 4,6 & 0,021 \\
\hline Feminino & 619 & 91,8 & 55 & 8,2 & \\
\hline \multicolumn{6}{|l|}{ Religião } \\
\hline Católica & 847 & 93,8 & 56 & 6,2 & NS \\
\hline Outras & 216 & 91,9 & 21 & 8,1 & \\
\hline \multicolumn{6}{|c|}{ Tipo de Escola } \\
\hline Pública & 543 & 91,7 & 49 & 8,3 & 0,028 \\
\hline Particular & 542 & 95,0 & 28 & 5,0 & \\
\hline
\end{tabular}

Obs.: Percentuais calculados sobre o total de casos com valor para cada variável. NS = sem diferença estatisticamente significativa. pela concentração de estudantes acima de 25 anos apenas nas primeiras. Esta diferença persistiu mesmo quando analisados apenas os estudantes com idade inferior a vinte anos, quando as idades médias foram de 17,8 e 16,7 anos, respectivamente.

Na Tabela 1, enumeram-se algumas características sócio-demográficas e de consumo de álcool na amostra, observando-se predominância de estudantes do sexo feminino (58\%), de religião católica (79\%), com experiência prévia de consumo de bebidas al coólicas (93\%) e CAGE positivo em $8 \%$ dos casos.

A prevalência de referência a experiência prévia de ingesta alcoólica entre adolescentes foi superior aos $80 \%$ encontrados por Fourney et al. (1988); aos 71,5\% observados por Pechansky (1993) entre aqueles de 10 a 18 anos da zona urbana de Porto Alegre e aos 53\% evidenciados por Sanchéz \& Zavala (1986) entre jovens estudantes de 14 a 18 anos. Todos estes estudos identificando uma maior prevalência de experimentação entre estudantes do sexo masculino.

Especificamente quanto à experiência prévia com bebida alcoólica em algum momento de suas vidas, ocorreu uma maior prevalência entre estudantes de sexo masculino ( $p \varangle 0,021$ ) e aqueles procedentes de escolas privadas ( $p<0,028$ ), não havendo diferença estatisticamente significativa quanto à idade $(p=0,16)$ e à religião referida. Entretanto, entre os estudantes que nunca experimentaram bebidas alcoólicas, dois terços eram mulheres e provenientes de escola pública (Tabela 2).

$\mathrm{Na}$ Tabela 3, foram discriminadas al gumas características do consumo de álcool entre os estudantes que referiram experiência prévia com bebidas alcoólicas. Metade $(49,8 \%)$ dos estudantes referiram uma freqüência semanal de consumo, usualmente em festas (60\%) e em bares ( $17 \%)$, sendo a cerveja ( $82 \%$ ) e o vinho $(61 \%)$ as bebidas mais comumente consumidas.

A prevalência de CAGE positivo no total da amostra foi $8,3 \%, 11 \%$ entre estudantes do sexo masculino ( $p \varangle 0,02$ ), $9 \%$ entre os estudantes das escolas particulares e $10 \%$ entre os estudantes com religião não-católica (ambos sem diferença estatisticamente significativa). A idade média dos alunos segundo positividade ou não ao teste CAGE foi de 17,3 e 17,8 anos, respectivamente $(p \triangleleft 0,01)$.

A variável poder de consumo apresentou diferença estatisticamente significativa quanto ao tipo de escola $(p \varangle 0,001)$, sexo $(p \varangle 0,001)$ e positividade do teste CAGE $(p=0,045)$, como pode ser observado na Tabela 5. Com relação 
ao tipo de escola, Carmona et al. (1986) referiram um maior consumo entre estudantes provenientes de escolas com nível sócio-econômico superior, sugerindo que o consumo pode ter alguma relação com a facilidade em obter bebida alcoólica ou com padrões de consumo familiares ou de grupos de amigos. Os aspectos psicossociais do consumo de bebidas alcoólicas entre estudantes ou adolescentes foram discutidos por uma série de autores, entre estes McLaughlin et al. (1985) e Fourney et al. (1988), cujos achados identificam a importân-

Tabela 3

Consumo de álcool entre estudantes que já experimentaram bebida alcoólica $(n=1.085)$.

\begin{tabular}{lrr}
\hline Variáveis & $\mathrm{n}$ & $\%$ \\
\hline $\begin{array}{l}\text { Freqüência de consumo } \\
\text { de álcool }\end{array}$ & & \\
$\quad$ Semanalmente & 519 & 49,8 \\
Mensalmente & 304 & 29,1 \\
Anualmente & 196 & 18,7 \\
Diariamente & 25 & 2,4 \\
Local de consumo & & \\
mais freqüente & & \\
Em festas & 573 & 60,4 \\
Em bares & 164 & 17,0 \\
Em casa, com os pais & 118 & 12,4 \\
Na casa de amigos & 49 & 5,2 \\
Em casa, com amigos & 33 & 3,5 \\
Em casa, sozinho & 10 & 1,1 \\
Outros locais & 4 & 0,4 \\
Tipo de bebida consumida & & \\
Cerveja & 894 & 82,4 \\
Vinho & 658 & 60,6 \\
Coquetéis & 456 & 42,0 \\
Martini & 338 & 31,2 \\
Uísque & 326 & 30,0 \\
Vodka & 328 & 30,2 \\
Licores & 308 & 28,4 \\
Cachaça & 213 & 19,6 \\
Campari & 164 & 15,1 \\
Conhaque & & 10,3 \\
Onderberg & & \\
\hline
\end{tabular}

Obs.: Percentuais calculados sobre o total de casos com valor. cia do padrão de consumo dos pais nas faixas etárias mais precoces, além do fato de os estudantes costumarem ter sua primeira experiência com álcool no núcleo familiar. Mais para o final da adolescência, entretanto, o padrão de consumo dos amigos está mais relacionado com o padrão de consumo do adolescente. Confirmando estes achados, Azevedo et al. (1991), referindo-se a crianças menores de 15 anos de idade em região de colonização italiana, identificou a oferta de vinho pelos pais aos filhos desde os primeiros meses de vida, com mais

Tabela 4

Relação entre sexo, religião e tipo de escola com o resultado do teste CAGE.

\begin{tabular}{|c|c|c|c|c|c|}
\hline \multirow[t]{3}{*}{ Variáveis } & \multicolumn{4}{|c|}{ CAGE } & \multirow[b]{3}{*}{$p$} \\
\hline & \multicolumn{2}{|c|}{ Positivo } & \multicolumn{2}{|c|}{ Negativo } & \\
\hline & $n$ & $\%$ & $n$ & $\%$ & \\
\hline \multicolumn{6}{|l|}{ Sexo } \\
\hline Masculino & 50 & 10,6 & 423 & 89,4 & 0,02 \\
\hline Feminino & 44 & 6,7 & 612 & 93,3 & \\
\hline \multicolumn{6}{|l|}{ Religião } \\
\hline Católica & 69 & 7,8 & 814 & 92,2 & NS \\
\hline O utras & 23 & 10,0 & 207 & 90,0 & \\
\hline \multicolumn{6}{|l|}{ Escola } \\
\hline Pública & 45 & 7,9 & 527 & 92,1 & NS \\
\hline Particular & 49 & 8,7 & 513 & 91,3 & \\
\hline
\end{tabular}

Obs.: Percentuais calculados sobre o total de casos com valor para cada variável. NS $=$ sem diferença estatisticamente significativa.

Tabela 5

Relação entre sexo, tipo de escola e resultado do teste CAGE e posição sócio-econômica.

\begin{tabular}{lccc}
\hline Variáveis & \multicolumn{2}{c}{ Posição sócio-econômica } \\
& $n$ & média \pm DP & $p$ \\
\hline $\begin{array}{l}\text { Sexo } \\
\text { Masculino } \\
\text { Feminino }\end{array}$ & 480 & $24,29 \pm 9,78$ & $<0,0001$ \\
Escola & 675 & $21,60 \pm 9,59$ & \\
Pública & & & \\
Particular & 591 & $16,77 \pm 6,64$ & $<0,0001$ \\
CAGE & 567 & $28,95 \pm 8,49$ & \\
Negativo & & & 0,045 \\
Positivo & 1.031 & $22,71 \pm 9,74$ & \\
\end{tabular}

Obs.: Posição sócio-econômica = score médio dos bens referidos no questionário de aferição, calculados sobre o total de casos com valor.

Percentuais calculados sobre o total de casos com valor para cada variável. 
da metade (58\%) destas crianças tendo iniciado o uso de álcool antes da primeira década de vida.

Testando a hipótese inicial deste estudo, de que haveria uma relação entre o tipo de escola e o consumo de álcool, o que seria de se esperar, já que a variável mais discriminante neste estudo foi a de poder de consumo, não se encontrou associação estatisticamente significativa quanto à positividade do Teste CAGE, controlando-se para as variáveis sexo, idade e posição sócio-econômica $(p=0,14)$.

\section{Conclusão}

Apesar de reconhecimento do alcoolismo como prioridade em saúde pública e da difusão dos vários agravos gerados à saúde pela ingesta de álcool, que não apenas depreciam agudamente a qualidade de vida dos indivíduos com problemas de ingesta e dependência, mas podem culminar em uma redução média de 15 anos na expectativa de vida (Schuckit, 1991;

\section{Referências}

ALMEIDA, P. M. \& WICKERHAUSER, H., 1992. Quem é quem? Novos padrões classificam a riqueza do consumidor. Veja, 1.219(5):77.

AZEVEDO, L. B.; BOLZZONI, A.; SILVEIRA, T. R. \& SOIBELMAN, M., 1991. Consumo de bebidas alcoólicas por menores de 15 anos residentes em um município de colonização tipicamente italiana no Rio Grande do Sul. Revista da Associação Médica do Rio Grande do Sul, 35:15-18.

BERNARD, M. W.; MUNFORDT, J. \& MURRAY, R. M. A, 1984. Discriminal-Function Analysis of Screening Test for Excessive Drinking and Alcoholism. Journal of Studies on Alcohol., 45:81-86.

CARMONA A. E.; RODRIGUES, G.; ARDILES, J.; AGUILA, M.; GALLARDO, G. \& SANTANDER, D., 1986. Factores socioeconómicos y ingestión de alcohol en estudiantes secundários. Revista Médica do Chile, 114:474-482.

CONROY, R. W., 1988. The many facets of adolescents drinking. Bulletin of the Menninger Clinic, 52:189282.
Scmidst \& De Lint, 1972), verificou-se ainda uma prevalência preocupante de 8,3\% entre os estudante secundaristas. Mormente pelo fato de, apesar de os estudantes se encontrarem majoritariamente na faixa etária proibida de comprar bebidas alcoólicas, os achados apontam que o consumo destas ocorreu usualmente em festas ou bares.

Neste estudo não se encontraram diferenças significativas entre o tipo de escola freqüentada (pública ou privada), havendo concordância com os estudos que apontaram maiores prevalências entre o sexo masculino e níveis sócio-econômicos mais elevados, para a faixa etária em análise. Tendo em vista a alta morbimortalidade relacionada ao uso de álcool, enfatiza-se a necessidade de novos trabalhos nesta área, bem como o emprego efetivo pelos profissionais da área da saúde dos diferentes métodos de detecção de problemas relacionados ao uso de álcool, notadamente entre as faixas etárias mais precoces, casualmente as mais responsivas aos programas de prevenção.
DUNCAN, B. B., 1991. As Desigualdades Sociais da Distribuição de Riscos para Doenças Não-Transmissíveis. Tese de Doutorado, Porto Alegre: Faculdade de Medicina, Universidade Federal do Rio Grande do Sul.

EWING, J. A., 1984. Detecting al coholism: The CAGE questionnaire. Journal of the American Medical Association, 252:1905-1907.

FORNEY, F. D. \& PECHANSKI, F., 1992. Uso não médico de fármacos. In: Farmacologia Clínica: Fundamentos da Terapêutica Racional (F. D. Fuchs \& L. Wannamacher, orgs.), pp. 359-381, Rio de Janeiro: Guanabara-Koogan.

FUCHS, F. D. \& PECHANSKI, F., 1992. Uso não médico de fármacos. In: Farmacologia Clínica: Fundamentos da Terapêutica Racional (F. D. Fuchs \& L. Wannamacher, orgs.), pp. 359-381, Rio de Janeiro: Guanabara-Koogan.

GIGANTE, P. L., 1988. Alcoolismo eUso de Álcool. Relação com o Perfil Sócio-Econômico deAdultos Residentes em Porto Alegre. Tese de Doutorado, Porto Alegre: Faculdade de Medicina, Clínica Médica, Universidade Federal do Rio Grande do Sul. 
HAYS, J. T. \& SPICKART, 1987. Alcoholism: early diagnosis and intervention. Journal of General Internal Medicine, 2:420-427.

LOWINSON, J. H.; RUIZ, P. \& MILLMAN, R. B., 1992. Substance Abuse. A Compreensive Textbook.2nd ed., Baltimore: Williams \& Wilkins.

KNAPP, P.; BERTOLOTE, J. M.; WOITOWITZ, A. \& MONTI, M., 1994. Prevenção da Recaída. Um Manual para Pessoas com Problemas pelo Uso do Álcool eDrogas. Porto Alegre: Artes Médicas.

MAGRUDER-HABIB, K.; STEVENS, H. A. \& ALLING, W. C., 1993. Relative performance of the MAST, VAST and CAGE versus DSM-III-R Criteria for alcohol dependence. Journal of Clinical Epidemiology, 46:435-441.

MALBERGIER, A.; BRASILIANO, S. \& BETTARELLO, S. V., 1992. O alcoolismo na prática clínica. Revista Brasiliera de Clínica \& Terapêutica, 21:446-452.

MASUR, J.; CAPRIGLIONE, M. J.; MONTEIRO, M. G. \& JORGE, M. R., 1985. Detecção precoce do alcoolismo em clínica médica através do questionário CAGE: utilidade e limitações. Jornal Brasileiro de Psiquiatria, 34:31-34.

MASUR, J. \& MONTEIRO M. J., 1983. Validation of the "CAGE" alcoholism screening test in a Brazilian psychiatric inpatient hospital setting. Brazilian Journal of Medical and Biological Research, 16: 215-218.

MAYFIELD, D.; MCLEOD, G. \& HALL, P., 1974. The CAGE questionnaire: validation of a new alcoholism screening instrument. American Journal of Psychiatry, 131:1121-1123.

MCLAUGHLIN, R. J.; BAUER, P. E.; BURNSIDE, M. A. \& POKORNY, A. D., 1985. Psychosocial correlates of alcohol use at two age levels during adolescence. Journal of Studies on Al cohol, 46:212-218.

MOREIRA, L. B., 1993. Padrões de Emprego de Bebidas Alcoólicas em Porto Al egre: Estudo de Indicadores de Dependência, Abuso, Consumo de Risco e Fatores Associados em uma Amostra Representativa da Região Urbana. Dissertação de Mestrado, Porto Alegre: Faculdade de Medicina, Universidade Federal do Rio Grande do Sul.
ODERICH, G. S. C., PECHANSKY, F., TATSCH, F. F., CAVAZZOLA, L. T., BOENO, R. L. \& MENEGAZ, F., 1995. Consumo de bebidas alcoólicas em alunos de escolas públicas de Porto Alegre. Revista da Associação Médica do Rio Grande do Sul, 39:229-236.

PECHANSKY, F., 1993. O Uso de Bebidas Al coólicas em Adol escentes Resi dentes na Cidade de Porto Alegre: Características de Consumo eProblemas Associados. Dissertação de Mestrado, Porto Alegre: Faculdade de Medicina, Universidade Federal do Rio Grande do Sul.

ROYCE, J. E., 1981. Alcohol Problems and Alcoholism. New York: Free Press.

RUEFF, B.; CRNAC, J. \& DARNE, B., 1989. Dépistage de malades "alcooliques" par l'autoquestionnaire systématique DETA: parmi des consultants hospitaliers. La Presse Médicale, 18:1.654-1.656.

SANCHÉZ, M. \& ZAVALA, G. G., 1986. Estudio epidemiológico sobre el uso de alcohol en población joven de 14 a 18 años. Revista de Salud Pública, 28:371-379.

SCHUCKIT, M. A., 1991. Alcoolismo: uma introdução. In: Abuso de Álcool e Drogas - Uma Orientação Clínica ao Diagnóstico e Tratamento (M. A. Schuckit, org.), p. 356, Porto Alegre: Artes Médicas.

SCMIDST, W. \& DE LINT, J., 1972. Causes of death in alcoholics. Studies in Alcohol, 33:171-185.

SOIBELM AN, M. \& LUZ JR, E., 1996. Problemas relacionados ao consumo de álcool. In: Medicina Ambulatorial: Condutas Clínicas em Atenção Primária (B. B. Duncan; M. I. Schmidt \& E. R. J. Giugliani, orgs.), pp. 286-295, 2a ed., Porto Alegre: Artes Médicas.

URZUA, R. F. \& KAEM PFFER, A. M., 1985. Alcoholism in adolescence and youth: the chilean experience. The health of adolescents and the youth in the Americas. PanAmerican Health Organization Scientific Publication, no 489, pp. 174-186, Washington, D. C.:PAHO. 